\title{
Editorial for the Special Issue "Selected Papers from the 16th International Conference on Squeezed States and Uncertainty Relations (ICSSUR 2019)"
}

\author{
Luis L. Sánchez-Soto ${ }^{1, *}$ and Margarita A. Man'ko ${ }^{2, *}$ \\ 1 Departamento de Óptica, Facultad de Fisica, Universidad Complutense, 28040 Madrid, Spain \\ 2 Lebedev Physical Institute, Leninskii Prospect 53, 119991 Moscow, Russia \\ * Correspondence: lsanchez@ucm.es (L.L.S.-S.); mmanko@sci.lebedev.ru (M.A.M.)
}

Received: 28 August 2020; Accepted: 1 September 2020; Published: 2 September 2020

The first quantum revolution started in the early 20th century and gave us new rules that govern physical reality. Accordingly, many devices that changed dramatically our lifestyle, such as transistors, medical scanners and lasers, appeared in the market. This was the origin of quantum technology, which allows us to organize and control the components of a complex system governed by the laws of quantum physics. This is in sharp contrast to conventional technology, which can only be understood within the framework of classical mechanics.

We are now in the middle of a second quantum revolution. Although quantum mechanics is nowadays a mature discipline, quantum engineering as a technology is now emerging in its own right. We are about to manipulate and sense individual particles, measuring and exploiting their quantum properties. This is bringing major technical advances in many different areas, including computing, sensors, simulations, cryptography and telecommunications.

The present collection of selected papers, all of them being invited talks at the16th International Conference on Squeezed States and Uncertainty Relations (ICSSUR 2019), held in Madrid from 17 to 21 June 2019, is a clear demonstration of the tremendous vitality of the field. The issue is composed of contributions from world leading researchers in quantum optics and quantum information, and presents viewpoints, both theoretical and experimental, on a variety of modern problems. We are confident that the reader will enjoy the present collection; it will be useful to experts working in all branches of quantum science.

The contributions to this collection deal with the modern results obtained in connection with the development of quantum technologies based on quantum mechanics, quantum information and quantum optics, as well as a better understanding of the foundations of quantum physics. It contains contemporarytheory of quantum phenomena, including practical applications and discussions of measurement procedures; see the paper "On the Prospects of Multiport Devices for Photon-Number-Resolving Detection" by Teo, Y.S.; Jeong, H.; Řeháček, J.; Hradil, Z.; Sanchez-Soto, L.L.; Silberhorn, C. [1].

The important result is searching forand finding during the last decades the possibility of formulating the basic notion of the state of a quantum system, like an atom or spin system, conventionally presented in terms of the wave function or density matrix, by using the standard probability distribution of the classical-like random variables given in the paper "Probability Representation of Quantum Mechanics Where System States Are Identified with Probability Distributions" by Chernega, V.N.; Man'ko, O.V.; Man'ko, V.I. [2]. The result is based on the existence of invertible maps of the vectors and density operators acting in Hilbert spaces onto the probability distributions.

The photon states, which in the previous period of quantum optics development were associated with quasidistributions like Wigner functions, Husimi-Kano functions and Glauber-Sudarshan functions, were found to be associated with the tomographicprobability distributions completely 
describing the states of quantum electromagnetic fields. An analogous association was also established for spin states and N-level atom states. In connection with discovering the possibility of describing quantum states by probability distributions, the superposition principle of quantum states responsible for the interference phenomenon was found to be formulated in terms of the nonlinear addition rule of probability distributions providing the probability distribution of the resulting state; see "Superposition Principle and Born's Rule in the Probability Representation of Quantum States" by Doskoch, I.Ya.; Man'ko, M.A. [3].

The other important development of quantum theory connected with the employment of coherent states and properties of oscillators, including their behavior in the presence of non-Hermitian Hamiltonians, is discussed in papers "Nonclassical States for Non-Hermitian Hamiltonians with the Oscillator Spectrum" by Zelaya, K.; Dey, S.; Hussin, V.; Rosas-Ortiz, O. [4], and “Coherent States for the Isotropic and Anisotropic 2D Harmonic Oscillators" by Moran, J.; Hussin, V. [5].

A recently developed group and graph theoretical approach aiming at the construction of large sets of mutually unbiased bases in finite-dimensional Hilbert spaces is discussed in the paper "Mutually Unbiased Bases and Their Symmetries" by Alber, G.; Charnes, C. [6]. In this approach, the construction of mutually unbiased bases in a Hilbert space of given dimension is reformulated as a clique-finding problem of a Cayley graph associated with a finite basis group. This approach offers the possibility to enlarge and possibly also to complete already known systems of mutually unbiased basis systems.

This collection of papers contains also the development of the theory of classical mechanics and relativistic dynamics, and its relation with the Poincare group; see the paper "Descriptions of Relativistic Dynamics with World Line Condition" by Ciaglia, F.M.; Di Cosmo, F.; Ibort, A.; Marmo, G. [7].

Furthermore, group theory, and oscillator creation and annihilation operator algebra, discussed in connection with Einstein's formula relating the particle energy with its mass and light velocity, are given in the contribution "Einstein's $E=m c^{2}$ Derivable from Heisenberg's Uncertainty Relations" by Başkal, S.; Kim, Y.S.; Noz, M.E. [8]. In this connection of classical mechanics with the quantum approach, one can point out that the probability distributions identified with quantum states obey kinetic equations of classical-like type, which presentother forms of the Schrödinger and von Neumann equations of the conventional formulation of quantum mechanics.

The phenomena of quantum correlations, including entanglement and its characteristics, are reviewed in detail in the following papers: "Distance between Bound Entangled States from Unextendible Product Bases and Separable States" by Wieaniak, M.; Pandya, P.; Sakarya, O.; Woloncewicz, B. [9] and "Selective Engineering for Preparing Entangled Steady States in Cavity QED Setup" by Sousa, E.H.S.; Roversi, J.A. [10]. Entanglement is considered for different kinds of quantum systems, both for systems with continuous variables and systems with discrete variables. The properties of nonclassical states of light are discussed in paper "Resource Theories of Nonclassical Light" by Tan, K.C.; Jeong, H. [11].

New important results describing the behavior of charged particles moving in the time-dependent magnetic field in solenoid are presented for new kinds of solenoid forms and different time-dependencies of magnetic fields; see the paper "A Quantum Charged Particle under Sudden Jumps of the Magnetic Field and Shape of Non-Circular Solenoids" by Dodonov, V.V.; Horovits, M.B. [12].

It should be pointed out that here one can find biographic memorial details of Roy Glauber and George Sudarshan, pioneers of quantum optics, and a kaleidoscope of the ICSSUR participants during the last three decades, presented in the above-mentioned contribution [3]. The scientific collaboration of George Sudarshan with friends and colleagues from European Universities and theirfundamental results in quantum optics, quantum mechanics and quantum field theory are reviewed in the paper "Remembering George Sudarshan" by Ibort, A.; Marmo, G. [13].

We hope that this collection of papers will be useful to get new results to be discussed at the next meeting of the ICSSUR series. This event started in 1991 as the Joint Seminar on Squeezed States and Uncertainty Relations of the University of Maryland at College Park and the Lebedev Physical 
Institute in Moscow, with Young Suh Kim and Vladimir I. Man'ko as its Founders; later on, it was converted into the International Conference on Squeezed States and Uncertainty Relations, which takes place over the world every two years; see https:/www.mdpi.com/journal/quantumrep/special_ issues/quantumrep_ICSSUR2019.

Funding: This research received no external funding.

Conflicts of Interest: The authors declare no conflict of interest.

\section{References}

1. Teo, Y.S.; Jeong, H.; Řeháček, J.; Hradil, Z.; Sánchez-Soto, L.L.; Silberhorn, C. On the Prospects of Multiport Devices for Photon-Number-Resolving Detection. Quantum Rep. 2019, 1, 15. [CrossRef]

2. Chernega, V.N.; Man'ko, O.V.; Man'ko, V.I. Probability Representation of Quantum Mechanics Where System States Are Identified with Probability Distributions. Quantum Rep. 2020, 2, 6. [CrossRef]

3. Doskoch, I.Y.; Man'ko, M.A. Superposition Principle and Born's Rule in the Probability Representation of Quantum States. Quantum Rep. 2019, 1, 13. [CrossRef]

4. Zelaya, K.; Dey, S.; Hussin, V.; Rosas-Ortiz, O. Nonclassical States for Non-Hermitian Hamiltonians with the Oscillator Spectrum. Quantum Rep. 2020, 2, 2. [CrossRef]

5. Moran, J.; Hussin, V. Coherent States for the Isotropic and Anisotropic 2D Harmonic Oscillators. Quantum Rep. 2019, 1, 23. [CrossRef]

6. Alber, G.; Charnes, C. Mutually Unbiased Bases and Their Symmetries. Quantum Rep. 2019, 1, 20. [CrossRef]

7. Ciaglia, F.M.; di Cosmo, F.; Ibort, A.; Marmo, G. Descriptions of Relativistic Dynamics with World Line Condition. Quantum Rep. 2019, 1, 16. [CrossRef]

8. Başkal, S.; Kim, Y.S.; Noz, M.E. Einstein's $\mathrm{E}=\mathrm{mc}^{2}$ Derivable from Heisenberg's Uncertainty Relations. Quantum Rep. 2019, 1, 21. [CrossRef]

9. Wieśniak, M.; Pandya, P.; Sakarya, O.; Woloncewicz, B. Distance between Bound Entangled States from Unextendible Product Bases and Separable States. Quantum Rep. 2020, 2, 4. [CrossRef]

10. Sousa, E.H.S.; Roversi, J.A. Selective Engineering for Preparing Entangled Steady States in Cavity QED Setup. Quantum Rep. 2019, 1, 7. [CrossRef]

11. Tan, K.C.; Jeong, H. Resource Theories of Nonclassical Light. Quantum Rep. 2019, 1, 14. [CrossRef]

12. Dodonov, V.V.; Horovits, M.B. A Quantum Charged Particle under Sudden Jumps of the Magnetic Field and Shape of Non-Circular Solenoids. Quantum Rep. 2019, 1, 17. [CrossRef]

13. Ibort, A.; Marmo, G. Remembering George Sudarshan. Quantum Rep. 2019, 1, 24. [CrossRef]

(C) 2020 by the authors. Licensee MDPI, Basel, Switzerland. This article is an open access article distributed under the terms and conditions of the Creative Commons Attribution (CC BY) license (http://creativecommons.org/licenses/by/4.0/). 